[Article]

\title{
液态聚乙二醇 $\mathrm{CH}_{2}$ 剪切振动和扭转振动 一拉曼光谱和密度泛函理论计算
}

\author{
韩 否 ${ }^{1}$ 彭 丽 $^{1}$ 蔡凌云 ${ }^{1}$ 郑旭明 ${ }^{1, *} \quad$ 张富山 $^{2}$
}

( ${ }^{1}$ 浙江理工大学化学系, 杭州 310018; 2 恒安集团技术中心, 福建 晋江 362261)

\begin{abstract}
摘要: 提出了一种新方案, 以有效洞察液态中共存构象异构体对聚氧乙烯(PEO)实际振动光谱的贡献。通过 定义 6 种一 $\left(\mathrm{CH}_{2} \mathrm{CH}_{2} \mathrm{O}\right)$ 一重复单元构象, 构建并优化得到4种全同 $\mathrm{EO}$ 构象组合 $\left[(T G T)_{10} \text { 、(TTT }\right)_{10}$ 、(TTG) 10 和 $(G T G)_{10}$ 和 3 种其它 EO 构象组合的构象异构体结构, 并在 PCM 溶剂模型和 B3LYP/6-31G $(d)$ 理论水平下, 对 结构进行了优化和振动频率计算。通过振动模式分析, 提出描述 PEO400 各构象异构体的不同 $\mathrm{CH}_{2}$ 剪切振动 和 $\mathrm{CH}_{2}$ 扭转振动模式的统一标准, 确定了 4 种 $\mathrm{CH}_{2} \mathrm{CH}_{2}-\mathrm{O}-\mathrm{CH}_{2} \mathrm{CH}_{2}$ 链段构象和相关振动模式, 以及它们与 振动频率大小的关系, 并应用于实际拉曼光谱的指认。
\end{abstract}

关键词：聚氧乙烯; 构象; 拉曼光谱; 密度泛函理论计算

中图分类号: 0641

\section{$\mathrm{CH}_{2}$ Scissor and Twist Vibrations of Liquid Polyethylene Glycol Raman Spectra and Density Functional Theory Calculations}

\author{
HAN Lei ${ }^{1} \quad$ PENG Li ${ }^{1} \quad$ CAI Ling-Yun ${ }^{1} \quad$ ZHENG Xu-Ming ${ }^{1, *} \quad$ ZHANG Fu-Shan ${ }^{2}$ \\ ('Department of Chemistry, Zhejiang Sci-Tech University, Hangzhou 310018, P. R. China; \\ ${ }^{2}$ Technology Center, Hengan Group, Jinjiang 362261, Fujian Province, P. R. China)
}

\begin{abstract}
A new method is developed to effectively study the contributions of various coexisting conformations to the actual vibrational spectrum of liquid polyethylene oxide (PEO). By defining six conformations for the $-\left(\mathrm{CH}_{2} \mathrm{CH}_{2} \mathrm{O}\right)$ - unit, four isomers from the combinations of all the same EO conformations [(TGT) ${ }_{10}$, (TTT $)_{10}$, $(\mathrm{TTG})_{10}$, and $\left.(\mathrm{GTG})_{10}\right]$ and three isomers from the combinations of other EO conformations were constructed. Their optimized geometric structures and the corresponding vibrational frequencies were then computed. The unified standards that describe the different types of the $\mathrm{CH}_{2}$ scissor and $\mathrm{CH}_{2}$ twist vibrational modes for PEO400 are proposed through the analysis of the normal modes. The relationships between the four $\mathrm{CH}_{2} \mathrm{CH}_{2}-\mathrm{O}-$ $\mathrm{CH}_{2} \mathrm{CH}_{2}$ conformations and the various $\mathrm{CH}_{2}$ scissor and $\mathrm{CH}_{2}$ twist vibrational modes and frequencies are determined, and the results are used to assign the practical vibrational spectra.
\end{abstract}

Key Words: Polyethylene oxide; Conformation; Raman spectrum; Density functional theory calculation

\section{1 引言}

以氧乙烯基(EO)为重复单元的聚氧乙烯(PEO) 因自身的亲水性而常用作两亲分子的亲水端。
如，烷基聚氧乙烯醚非离子性表面活性剂和聚氧 乙烯聚氧丙烯醚嵌段共聚物中的氧乙烯基。聚氧 乙烯基(分子)主链中 $-\mathrm{CH}_{2}-\mathrm{O}-、-\mathrm{CH}_{2}-\mathrm{CH}_{2}-$

Received: November 28, 2016; Revised: January 21, 2017; Published online: February 8, 2017.

${ }^{*}$ Corresponding author. Email: zxm@zstu.edu.cn; Tel: +86-571-86843699.

The project was supported by the National Natural Science Foundation of China (21473163) and National Basic Research Program of China (973) (2013CB834604). 
等结构单元的旋转使单条 PEO 分子的构象稳定性 会随外部因素(相态、温度、溶剂、离子)等的变化 而变化。因此, PEO 的理化和应用性能与聚合度 $m$ 和 EO 空间取向等密切相关。

晶态 PEO 的红外光谱、拉曼光谱和 X 射线衍 射谱的研究结果显示, PEO 分子链为螺旋(helix)结 构, 7 个 $\mathrm{EO}$ 单元形成 2 个完整螺旋(简称 $7 / 2$ 螺 旋 $)^{1-4}$ 。其中, 绕 $\mathrm{O}-\mathrm{CH}_{2}, \mathrm{CH}_{2}-\mathrm{CH}_{2}$, 和 $\mathrm{CH}_{2}-\mathrm{O}$ 单键的内旋转构象分别为反式构象 $(\mathrm{T})$, 偏转构象 (G) 和 T(简称 TGT 构象) ${ }^{5}$ 。以 TGT 为重复单元的螺 旋链具有两个二重对称轴, 一个轴穿过氧原子, 另一轴平分 $\mathrm{C}-\mathrm{C}$ 键。 $\mathrm{X}$ 射线衍射光谱显示, $\mathrm{PEO}$ 晶体的单位晶包属于单斜晶系, 空间群为 $P 2{ }_{1} / a$, 内含四条螺旋分子链 ${ }^{6}$ 。在过去 50 多年里, $(\mathrm{TGT})_{m}$ 构象在解释晶态 PEO 结构与物性关系中发挥着关 键作用。例如, 基于 $(\mathrm{TGT})_{m}$ 的简正振动计算频率 能与常温晶态 $\mathrm{PEO}$ 实验振动频率吻合 $1,2,7,8$ 。(TGT $)_{m}$ 构象异构体的链变形振动和 $\mathrm{C}-\mathrm{O}$ 内旋转运动得到 低温远红外和拉曼光谱的印证 ${ }^{9}$ 。

与晶态 PEO 不同, 液态纯 PEO 分子链的晶格 堆积效应消失, 主链单键旋转产生大量构象异构 体共存。但迄今为止, 液态 PEO 的光谱研究一直 沿用 $(\mathrm{TGT})_{m}$ 构象 ${ }^{3,10}$ 。例如, 水溶液中 PEO 与水的 氢键作用 ${ }^{3}$, 液态或水溶液中 PEO 的结构有序 性 ${ }^{10}$, 水溶液中 PEO 与 $\mathrm{M}^{n+}$ 相互作用 ${ }^{4,11-17}$, 温度和 $\mathrm{H}_{2} \mathrm{O}$ 量对 $-\mathrm{C}-\mathrm{C}-(\mathrm{G}$ 构象 $)$ 和 $-\mathrm{C}-\mathrm{O}-(\mathrm{T}$ 构象 $)$ 结 构的影响等都只考虑 $(\mathrm{TGT})_{m}$ 构象 ${ }^{18}$, 而其它构象的 作用一概被忽略。究其原因, 主要是频率相近的 振动峰的叠加使液态 PEO 的振动光谱较晶态时大 为展宽, 谱带指认变得相当困难, 阐明其它构象 作用的相关研究相当稀少。为此, 本文采用密度 泛函理论计算方法, 结合拉曼光谱实验手段, 研 究常温下液态 PEO400 构象异构体的结构和振动光 谱。

\section{2 实验和理论计算}

\section{1 试剂与仪器}

\subsection{1 实验药品}

平均分子量 400 聚乙二醇(PEO400), 化学结构 式 $\mathrm{HO}-\left(\mathrm{CH}_{2} \mathrm{CH}_{2} \mathrm{O}\right)_{m}-\mathrm{H}(m=10)$, (纯度 $99.0 \%$, Sigma-Aldrich 公司); 平均分子量 1500 聚乙二醇 (PEO1500), 化学结构式 $\mathrm{HO}-\left(\mathrm{CH}_{2} \mathrm{CH}_{2} \mathrm{O}\right)_{m}-\mathrm{H}$ $(m=34)$, (纯度 $99.0 \%$, Sigma-Aldrich 公司)。

\section{1 .2 实验仪器}

傅里叶变换红外光谱仪 (Nicolet $57000.09 / \mathrm{cm}$, 美国尼高力仪器公司), 显微拉曼光谱仪 (*/PI Action Tri Vista，美国 Princeton Instruments Inc)。

\section{2 实验方法}

\subsection{1 傅里叶变换红外(FT-IR) 光谱实验}

实验使用 Nicolet $57000.09 / \mathrm{cm}$ 傅里叶红外光谱 仪。用毛细管蘸取少量液态的样品滴在 $\mathrm{KBr}$ 窗片 上, 并用另一片 $\mathrm{KBr}$ 窗片盖在上面, 使液膜均匀 分布待测; 实验扫描空气作为背景, 扫描次数 32 次, 扫描波数范围 $4000-400 \mathrm{~cm}^{-1}$ 。

\subsection{2 显微拉曼光谱}

实验使用*/PI Action Tri Vista 显微拉曼光谱仪 (micro-Raman)。实验样品直接放进比色㿼(10 mm $\times$ $10 \mathrm{~mm}, 2.5 \mathrm{~mL}$ )中, 在 $488 \mathrm{~nm}$ 激光波长下测定, 为 尽可能提高信噪比, 每张光谱大约采集 20-200 s。

\section{3 理论计算}

分子几何结构优化和振动频率计算在 B $3 \mathrm{LYP} /$ 6-31G $(d)$ 理论水平下获得, 所有量子化学计算均由 Gaussian 09W 程序包完成。

\section{3 结果和讨论}

\section{1 构象结构}

PEO 分子主链的单键旋转产生构象异构体。 按现行标记方法 ${ }^{18}$ 和分子式 $\mathrm{HO}\left(\mathrm{CH}_{2} \mathrm{CH}_{2} \mathrm{O}\right)_{10} \mathrm{H}$ 顺 序, 图 1 给出 6 种 $\mathrm{EO}$ 构象标记, 其中, 数字 $1 、 2$ 和 3 分别标记 $\mathrm{O}-\mathrm{C}, \mathrm{C}-\mathrm{C}$ 和 $\mathrm{C}-\mathrm{O}$ 单键。 $\mathrm{TTT}$ 和 $\mathrm{TGT}$ 等名称中, 反式构象 $\mathrm{T}$ 或扭转构象 $\mathrm{G}$ 表示以某 个键(如标记 2 的 $\mathrm{C}-\mathrm{C}$ 键)为中心的前后 2 个键(如 标记 1 的 $\mathrm{O}-\mathrm{C}$ 单键和标记 3 的 $\mathrm{C}-\mathrm{O}$ 键) 的相对空 间位置。表 1 分别给出了 $\mathrm{C}-\mathrm{O}-\mathrm{C}-\mathrm{C} 、 \mathrm{O}-\mathrm{C}-$

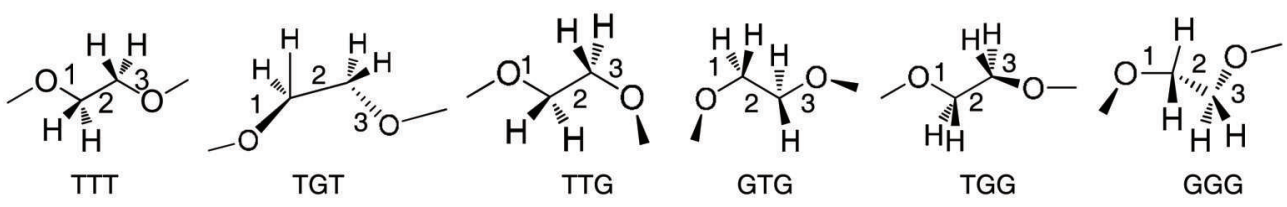

图 $1-\left[\mathrm{CH}_{2} \mathrm{CH}_{2} \mathrm{O}\right]$-单元的六种构象

Fig.1 Six conformations of the $-\left[\mathrm{CH}_{2} \mathrm{CH}_{2} \mathrm{O}\right]-$ unit 
$\mathrm{C}-\mathrm{O}$ 和 $\mathrm{C}-\mathrm{C}-\mathrm{O}-\mathrm{C}$ 所形成的二面角数据, 用以 描述各构象的相对空间位置。以这 6 种 $\mathrm{EO}$ 构象为 单元，构建了 6 种全同 $\mathrm{EO}$ 构象组合和 3 种随机 $\mathrm{EO}$ 构象组合的 PEO400构象异构体，并采用密度泛函 理论对 9 种初始结构进行结构优化。(TGG) $)_{10}$ 和 $(\mathrm{GGG})_{10}$ 构象异构体未能优化得到, 主要原因是两 个连续 $\mathrm{GG}$ 构象会导致 $\mathrm{OH}$ 基和 $\mathrm{CH}_{2}$ 基上两个 $\mathrm{H}$ 原 子在空间产生排斥力而不利于相关构象的稳定。
穷尽不同构象异构体来阐明构象与特征振动 频率大小的定量关系对构象数很有限的小分子体 系是一种常规策略，已广泛用于光谱指认中。但 这一策略对 EO 数较大的 PEO体系不适合, 因为其 构象数随 $\mathrm{EO}$ 数的增加呈几何级数增加。因此, 在 有限的计算成本中必须避开穷尽 PEO 全部构象异 构体的做法。

本文发现，决定 $\mathrm{CH}_{2}$ 剪式振动和 $\mathrm{CH}_{2}$ 扭转振动

表 1 B3LYP/6-31G $(d)$ 计算水平下获得的 7 种 PEO400 几何构象的能量和二面角

Table 1 Seven geometric conformations of PEO400 and the corresponding dihedral angles computed using B3LYP/6-31G(d) level of theory

\begin{tabular}{|c|c|c|c|c|c|}
\hline & Conformation & $-E /$ hatree & Zero-point vibrational energy $/\left(\mathrm{kJ} \cdot \mathrm{mol}^{-1}\right)$ & $\Delta E /\left(\mathrm{kJ} \cdot \mathrm{mol}^{-1}\right)^{\mathrm{a}}$ & Dihedral angles $/\left({ }^{\circ}\right)^{b}$ \\
\hline \multirow[t]{7}{*}{ gas phase } & $(\mathrm{TTT})_{10}$ & 1614.7124 & 1683.9310 & 0.0 & $180 / 180 / 180$ \\
\hline & $(\mathrm{TGT})_{10}$ & 1614.7069 & 1684.3496 & $14.65(14.65)$ & $177 / 72 / 177$ \\
\hline & $(\mathrm{TTG})_{10}$ & 1614.6948 & 1687.6991 & $46.47(50.24)$ & $177 / 177 / 82$ \\
\hline & $(\mathrm{GTG})_{10}$ & 1614.6836 & 1689.3738 & $75.78(80.81)$ & $92 / 177 / 92$ \\
\hline & (GTT-TTT-TTG-TTT) $)_{2}$-GTT-TTT & 1614.7045 & 1686.4430 & $20.93(23.45)$ & \\
\hline & $(\mathrm{GTT})_{2}(\mathrm{TTG})_{2}(\mathrm{GTT})_{2}(\mathrm{TTG})_{2}(\mathrm{GTT})_{2}$ & 1614.6964 & 1687.6991 & $41.87(45.22)$ & \\
\hline & $(\mathrm{GTG})_{6}(\mathrm{GTT})_{2}(\mathrm{GTG})_{2}$ & 1614.6862 & 1688.9551 & $69.08(74.11)$ & \\
\hline \multirow[t]{7}{*}{ water solvent model } & $(\mathrm{TGT})_{10}$ & 1614.7431 & 1685.1870 & 0 & $180 / 69 / 180$ \\
\hline & $(\mathrm{TTT})_{10}$ & 1614.7325 & 1681.4189 & $27.63(23.86)$ & $180 / 180 / 180$ \\
\hline & $(\mathrm{TTG})_{10}$ & 1614.7179 & 1686.4430 & $66.15(67.41)$ & $175 / 175 / 81$ \\
\hline & $(\mathrm{GTG})_{10}$ & 1614.7106 & 1689.3738 & $85.41(89.60)$ & $91 / 179 / 91$ \\
\hline & $(\text { GTT-TTT-TTG-TTT })_{2}$-GTT-TTT & 1614.7259 & 1684.3496 & $45.22(44.80)$ & \\
\hline & $(\mathrm{GTT})_{2}(\mathrm{TTG})_{2}(\mathrm{GTT})_{2}(\mathrm{TTG})_{2}(\mathrm{GTT})_{2}$ & 1614.7192 & 1686.0244 & $62.80(63.64)$ & \\
\hline & $(\mathrm{GTG})_{6}(\mathrm{GTT})_{2}(\mathrm{GTG})_{2}$ & 1614.7121 & 1688.5364 & $81.64(85.41)$ & \\
\hline
\end{tabular}

${ }^{a}$ energy difference corrected by zero-point vibrational energy;

${ }^{b}$ dihedral angles corresponding to $\mathrm{C}-\mathrm{O}-\mathrm{C}-\mathrm{C}, \mathrm{O}-\mathrm{C}-\mathrm{C}-\mathrm{O}$ and $\mathrm{C}-\mathrm{C}-\mathrm{O}-\mathrm{C}$, respectively

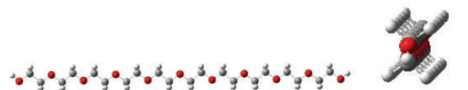

$(\mathrm{TTT})_{10}$

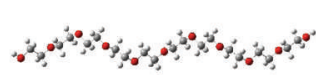

$(\mathrm{TTG})_{10}$<smiles>C#CC#CCCCCCC#CC=C</smiles>

$(\mathrm{TGT})_{10}$

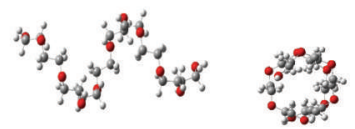

$(\mathrm{GTG})_{10}$

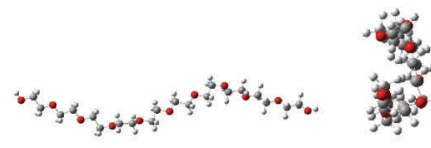

(GTT-TTT-TTG-TTT) ${ }_{2}$-GTT-TTT (A)

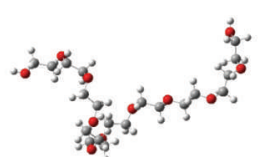

$(\mathrm{GTG})_{6}(\mathrm{GTT})_{2}(\mathrm{GTG})_{2}$ (C)

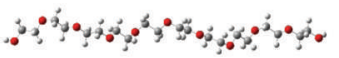

$(\mathrm{GTT})_{2}(\mathrm{TTG})_{2}(\mathrm{GTT})_{2}(\mathrm{TTG})_{2}(\mathrm{GTT})_{2}(\mathrm{~B})$

图 2 PCM 溶剂模型和 B3LYP/6-31G $(d)$ 计算水平下获得的 7 种代表性 PEO400 构象结构图

Fig.2 Seven conformational structures of PEO400 obtained using the PCM solvent model at B3LYP/6-31G(d) level of theory 


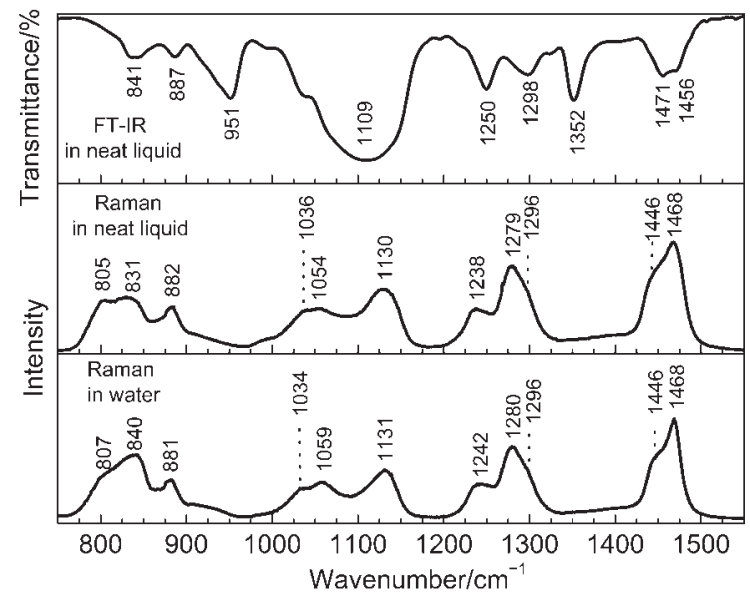

图 3 纯 PEO400 液体的傅里叶变换红外光谱(上)和拉曼光 谱(中)以及 $n\left(\mathrm{PEO400):} n\left(\mathrm{H}_{2} \mathrm{O}\right)=1: 1\right.$ 样品的拉曼光谱(下)

Fig.3 FT-IR (top) and Raman (middle) spectra of neat liquid PEO400, and Raman spectrum (bottom) of $n\left(\mathrm{PEO400)}: \boldsymbol{n}\left(\mathrm{H}_{2} \mathrm{O}\right)=1: 1\right.$ sample Raman excitation wavelength $=488 \mathrm{~nm}$

频率大小的关键因素是 $\mathrm{CH}_{2} \mathrm{CH}_{2}-\mathrm{O}-\mathrm{CH}_{2} \mathrm{CH}_{2}$ 链段 的构象及其振动模式(详见本文 2.3 节), 当(TGT) 10 构象异构体被确定为主要成分时, 只要 $\mathrm{CH}_{2} \mathrm{CH}_{2}-$ $\mathrm{O}-\mathrm{CH}_{2} \mathrm{CH}_{2}$ 链段的构象及其振动模式穷尽了，就 无需考虑更多其它 EO构象组合的构象异构体的数
量问题。图 2 示出 $(\mathrm{TTT})_{10}$ 、 $(\mathrm{TGT})_{10}$ 、 $(\mathrm{TTG})_{10}$ 、 $(\mathrm{GTG})_{10} 、(\mathrm{GTT}-\mathrm{TTT}-\mathrm{TTG}-\mathrm{TTT})_{2}$-GTT-TTT、 $(\mathrm{GTG})_{6}$ $(\mathrm{GTT})_{2}(\mathrm{GTG})_{2}$ 和 $(\mathrm{GTT})_{2}(\mathrm{TTG})_{2}(\mathrm{GTT})_{2}(\mathrm{TTG})_{2}(\mathrm{GTT})_{2}$ 等 7 种优化构象结构, 它们已包含了全部 4 种 $\mathrm{CH}_{2} \mathrm{CH}_{2}-\mathrm{O}-\mathrm{CH}_{2} \mathrm{CH}_{2}$ 链段的构象和振动模式。表 1 列出它们的能量和部分二面角数据。由表 1 可 见, 真空和气相时, (TTT $)_{10}$ 构象异构体的能量最 低, 稳定性依次为 $(\mathrm{TTT})_{10}>(\mathrm{TGT})_{10}>(\mathrm{TTG})_{10}>$ $(\mathrm{GTG})_{10}$; 而水溶剂模型时, $(\mathrm{TGT})_{10}$ 构象能量最 低, 稳定性依次为 $(\mathrm{TGT})_{10}>(\mathrm{TTT})_{10}>(\mathrm{TTG})_{10}>$ $(\mathrm{GTG})_{10}$ 。可见, 引入水溶剂的极性效应, $(\mathrm{TGT})_{10}$ 和(TTT $)_{10}$ 的稳定性互换位次。由表 1 可见, 3 种非 全同 EO 构象组合的 PEO400构象异构体的能量介 于 $(\mathrm{TGT})_{10}$ 和(GTG) $)_{10}$ 之间。

\section{2 振动光谱}

纯 PEO400 的液态傅立叶变换红外光谱和拉曼 光谱, 以及 $\mathrm{PEO} 400: \mathrm{H}_{2} \mathrm{O}=1: 1$ 样品的拉曼光谱示 于图 3。图 3 可见, 纯 PEO400 的液态拉曼谱带较 晶态时要宽得多, 预示有多种构象异构体共存。 同时，水与 PEO400 的氢键相互作用对 500-1600 $\mathrm{cm}^{-1}$ 频率区域的拉曼光谱强度模式影响不大，但会 引起 830 和 $1238 \mathrm{~cm}^{-1}$ 等振动频率的些许位移。

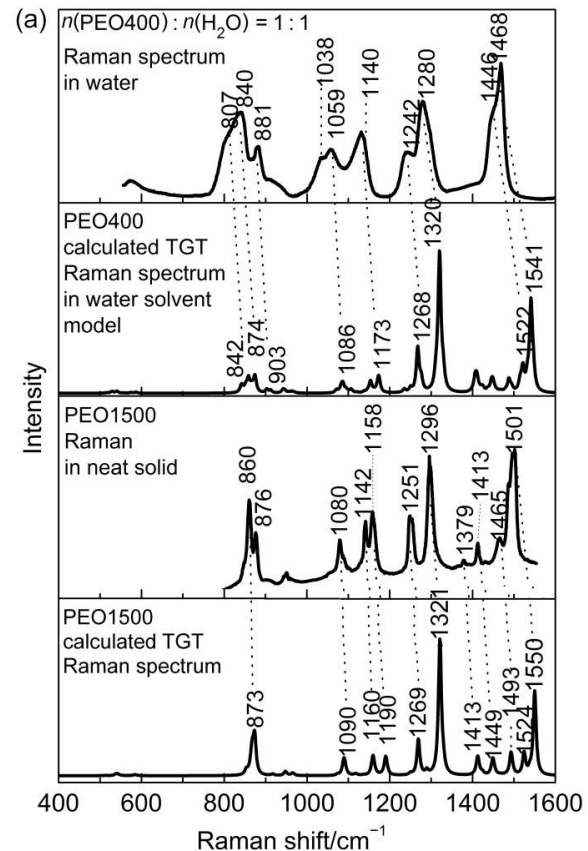

(b)

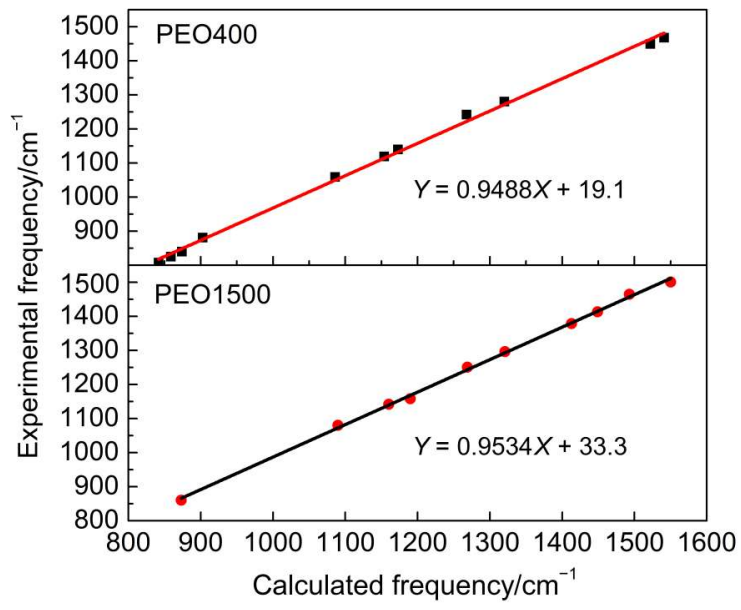

图 4 (a) B3LYP/6-31G $(d)$ 理论水平下计算获得的 TGT 异构体拉曼光谱与样品实验拉曼光谱的比较和

(b)主要实验频率与计算频率的线性回归拟合

Fig.4 (a) Comparison between the experimental Raman spectrum of sample and the calculated TGT Raman spectrum using B3LYP/6-31G(d) level of theory and (b) linear regression fitting between main experimental Raman frequencies and 
为了验证密度泛函理论计算在预测聚氧乙烯 醚聚合物的振动频率时的可靠性, 开展了 (TGT) $)_{34}$ 拉曼光谱的计算和 PEO1500 实验拉曼光谱的测 量, 结果示于图 4(a), 其中虚线示出主要实验谱带 与 $(\mathrm{TGT})_{34}$ 计算光谱带的试探性关联。图 4(b)(下)示 出实验和理论计算光谱主要振动峰间的线性回归 拟合结果, 结果显示, 相关系数 $R=0.999$, 标准 偏差 $=6.99$, 两者吻合良好, 说明密度泛函理论计 算方法是可靠的。

采用 PCM 溶剂模型下的 B3LYP/6-31G $(d)$ 计算
水平对图 2 中 7 种构象异构体的优化结构进行了振 动频率计算。表 2 列出了(TGT) $)_{10}$ 构象异构体的计算 频率与 PEO400 液态实验频率的比较, 以及初步的 光谱指认。图 4(a)(上)展示(TGT) $)_{10}$ 的计算拉曼光谱 与 PEO400 实验拉曼光谱的比较, 图 4(b)(上)展示 了相关振动频率的线性回归拟合结果，相关系数 $R=0.998$, 标准偏差 $=11.5$ 。图 4 和表 1 、表 2 结果 显示, 能量最低的(TGT) $)_{10}$ 构象异构体的拉曼光谱 强度模式能与实验拉曼光谱大致吻合，表明溶液 中 $(\mathrm{TGT})_{10}$ 构象结构是 PEO400 的主要存在形式之

表 2 PCM 溶剂模型和 B3LYP/6-31G $(d)$ 计算水平下 $(\text { TGT })_{10}$ 构象异构体的计算振动频率、PEO400 实验振动频率和光谱指认

Table 2 Calculated vibrational frequencies of (TGT) $)_{10}$ conformation using PCM solvent model and B3LYP/6-31G $(d)$ level of theory, the experimental frequencies and the assignments of PEO400

\begin{tabular}{|c|c|c|c|c|c|c|}
\hline & \multicolumn{2}{|l|}{ Calc. } & \multicolumn{2}{|c|}{ Raman } & \multirow{2}{*}{$\begin{array}{c}\text { FT-IR } \\
\text { neat liquid }\end{array}$} & \multirow{2}{*}{ Description } \\
\hline & $a($ Raman/IR $)$ & $b$ & neat liquid & water & & \\
\hline$v_{1}$ & $1541(139 / 0.1)$ & 1481 & $1468 \mathrm{~s}$ & $1468 \mathrm{~s}$ & $1471 \mathrm{w}$ & $\mathrm{CH}_{2}$ scissor \\
\hline$v_{2}$ & $1522(21.3 / 0.9)$ & 1463 & $1446 \mathrm{~m} \mathrm{sh}$ & $1446 \mathrm{~m} \mathrm{sh}$ & $1456 \mathrm{w}$ & \\
\hline$v_{3}$ & $1520(12.5 / 43.6)$ & 1456 & & & & \\
\hline$v_{4}$ & $1488(31.6 / 0.1)$ & 1431 & & & & $\mathrm{CH}_{2}$ wag \\
\hline$v_{5}$ & $1450(22.6 / 0.7)$ & 1393 & & & & \\
\hline$v_{6}$ & $1437(6.7 / 40.7)$ & 1383 & & & & $\mathrm{CH}_{2}$ wag $+\mathrm{H}-\mathrm{C}-\mathrm{O}-\mathrm{H}$ bend \\
\hline$v_{7}$ & $1424(10.1 / 3.4)$ & 1370 & & & $1352 \mathrm{~m}$ & $\mathrm{CH}_{2}$ wag \\
\hline$v_{8}$ & $1408(15.6 / 17.8)$ & 1355 & & & & \\
\hline$v_{9}$ & $1389(0.1 / 370)$ & 1337 & & & & \\
\hline$v_{10}$ & $1329(42.2 / 46.0)$ & 1280 & $1296 \mathrm{~m} \mathrm{sh}$ & $1296 \mathrm{~m} \mathrm{sh}$ & $1298 \mathrm{w}$ & $\mathrm{CH}_{2}$ twist $+\mathrm{H}-\mathrm{C}-\mathrm{O}-\mathrm{H}$ bend \\
\hline$v_{11}$ & $1320(171 / 2.9)$ & 1272 & $1279 \mathrm{~s}$ & $1280 \mathrm{~s}$ & $1250 \mathrm{~m}$ & $\mathrm{CH}_{2}$ twist \\
\hline$v_{12}$ & $1277(26.8 / 82.6)$ & 1231 & & & & \\
\hline$v_{13}$ & $1267(55.5 / 0.2)$ & 1221 & $1238 \mathrm{~m}$ & $1242 \mathrm{~m}$ & & \\
\hline$v_{14}$ & $1250(9.9 / 28.5)$ & 1206 & & & & $\mathrm{CH}_{2}$ twist $+\mathrm{H}-\mathrm{C}-\mathrm{O}-\mathrm{H}$ bend \\
\hline$v_{15}$ & $1235(8.3 / 28.7)$ & 1191 & & & & \\
\hline$v_{16}$ & $1172(14.3 / 147)$ & 1131 & $1130 \mathrm{~s}$ & $1131 \mathrm{~m}$ & & $\mathrm{C}-\mathrm{O}-\mathrm{C}$ asym. stretch \\
\hline$v_{17}$ & $1153(21.1 / 453)$ & 1113 & & & $1109 \mathrm{~s}$ & \\
\hline$v_{18}$ & $1135(0.04 / 889)$ & 1096 & & & & \\
\hline$v_{19}$ & $1111(1.0 / 39.9)$ & 1073 & & & & $\mathrm{CH}_{2}$ rock $+\mathrm{C}-\mathrm{O}$ stretch \\
\hline$v_{20}$ & $1106(7.4 / 28.5)$ & 1068 & & & & \\
\hline$v_{21}$ & $1100(1.8 / 68.4)$ & 1063 & & & & \\
\hline$v_{22}$ & $1088(13.8 / 156)$ & 1051 & $1054 \mathrm{~m}$ & $1059 \mathrm{~m}$ & $951 \mathrm{~m}$ & $\mathrm{C}-\mathrm{O}-\mathrm{C}$ sym. stretch \\
\hline$v_{23}$ & $1072(5.4 / 57.2)$ & 1036 & $1036 \mathrm{~m}$ & $1034 \mathrm{~m}$ & & $\mathrm{CH}_{2}$ rock $+\mathrm{H}-\mathrm{C}-\mathrm{O}-\mathrm{H}$ bend \\
\hline$v_{24}$ & $978(0.01 / 205)$ & 947 & & & & $\mathrm{C}-\mathrm{C}$ stretch \\
\hline$v_{25}$ & $969(1.4 / 27.1)$ & 938 & & & & \\
\hline$v_{26}$ & $964(3.4 / 53.4)$ & 934 & & & & \\
\hline$v_{27}$ & $952(0.4 / 42.2)$ & 922 & & & & \\
\hline$v_{28}$ & $942(7.5 / 13.5)$ & 913 & & & & \\
\hline$v_{29}$ & $912(5.7 / 35.6)$ & 884 & & & & $\mathrm{H}-\mathrm{C}-\mathrm{O}-\mathrm{H}$ bend \\
\hline$v_{30}$ & $903(8.3 / 26.8)$ & 876 & & & & \\
\hline$v_{31}$ & $874(33.4 / 0.03)$ & 848 & $882 \mathrm{~s}$ & $881 \mathrm{~m}$ & $887 \mathrm{w}$ & $\mathrm{CH}_{2}$ rock \\
\hline$v_{32}$ & $859(29.4 / 102)$ & 834 & $831 \mathrm{sh}$ & $840 \mathrm{~s}$ & $841 \mathrm{w}$ & \\
\hline$v_{33}$ & $842(9.1 / 4.9)$ & 818 & $805 \mathrm{~m} \mathrm{sh}$ & $806 \mathrm{~m} \mathrm{sh}$ & & \\
\hline
\end{tabular}

$a$ : B3LYP/6-31G $(d)$ calculated frequencies; $b$ : scaled frequencies using linear regression scaling method: Scaled $=0.9488 \times$ Calc. +19.07 ; sym: symmetric; asym: antisymmetric; s: strong; m: middle; w: weak; sh: shoulder 
一, 沿用 $(\mathrm{TGT})_{10}$ 构象指认液态拉曼光谱主要谱峰 是合理的, 但离全面阐述液态光谱仍有距离。例 如, $(\mathrm{TGT})_{10}$ 构象结构的计算拉曼光谱与实际光谱 的强度模式如 1446、1296、1131、1059、1034、 $881 、 840$ 和 $807 \mathrm{~cm}^{-1}$ 等的峰强度上, 存在较大差 异。部分原因可能是所采用的密度泛函理论计算 水平还不够高级, 使得 $(\mathrm{TGT})_{10}$ 的计算拉曼峰强度 与实际光谱有偏差, 但更主要的原因应该是其它 共存构象的叠加贡献。

\section{$3.3 \mathrm{CH}_{2} \mathrm{CH}_{2}-\mathrm{O}-\mathrm{CH}_{2} \mathrm{CH}_{2}$ 特征链段的振动模式}

\section{及其对实验光谱的贡献}

为了洞察不同 $\mathrm{CH}_{2} \mathrm{CH}_{2}-\mathrm{O}-\mathrm{CH}_{2} \mathrm{CH}_{2}$ 链段构象 的不同 $\mathrm{CH}_{2}$ 振动模式对实验光谱的贡献, 图 5 示出 由 PCM 溶剂模型和 B3LYP/6-31G $(d)$ 计算水平获得 的 7 种 PEO400 构象异构体的计算拉曼光谱。仔细 研究 1150-1600 $\mathrm{cm}^{-1}$ 光谱区域内各主要拉曼谱峰 的振动模式后发现, 它们可分别归类为 $\mathrm{CH}_{2} \mathrm{CH}_{2} \mathrm{OCH}_{2} \mathrm{CH}_{2}$ 链段 4 种构象的 $\mathrm{CH}_{2}$ 剪切振动和 $\mathrm{CH}_{2}$ 扭转振动, 具体见图 6 。相关的 $\mathrm{CH}_{2}$ 剪切振动 和 $\mathrm{CH}_{2}$ 扭转振动模式归纳如下。

(TGT) 10 构象异构体的 $\mathrm{CH}_{2} \mathrm{CH}_{2}-\mathrm{O}-\mathrm{CH}_{2} \mathrm{CH}_{2}$ 链 段为 GTTG 构象(简称 GTTG 链段, 下同)。因此, 1541、1522、1320 和 $1268 \mathrm{~cm}^{-1}$ 等 4 个拉曼峰都与 GTTG 构象有关。其中, $1541 \mathrm{~cm}^{-1}$ 峰为 $\mathrm{C}_{a} \mathrm{H}_{2} / \mathrm{C}_{a^{\prime}} \mathrm{H}_{2}$ 同向 $+\mathrm{C}_{\beta} \mathrm{H}_{2} / \mathrm{C}_{\beta^{\prime}} \mathrm{H}_{2}$ 同向及 $\mathrm{C}_{\alpha} \mathrm{H}_{2} / \mathrm{C}_{\beta} \mathrm{H}_{2}$ 背向 $+\mathrm{C}_{\alpha^{\prime}} \mathrm{H}_{2} / \mathrm{C}_{\beta^{\prime}} \mathrm{H}_{2}$ 背向剪切振动(简称 $\alpha / \alpha^{\prime}+\beta / \beta^{\prime}$ 两两同向 $\mathrm{CH}_{2}$ 剪切振 动, 下同); $1522 \mathrm{~cm}^{-1}$ 峰为 $\alpha / \beta+\alpha^{\prime} / \beta^{\prime}$ 两两同向 $\mathrm{CH}_{2}$ 剪切振动; $1320 \mathrm{~cm}^{-1}$ 峰为 $\alpha / \alpha^{\prime}+\beta / \beta^{\prime}$ 两两同向 $\mathrm{CH}_{2}$ 扭转(twist)振动; $1268 \mathrm{~cm}^{-1}$ 峰为 $\alpha / \beta+\alpha^{\prime} / \beta^{\prime}$ 两两同 向 $\mathrm{CH}_{2}$ 扭转振动。

(TTT $)_{10}$ 构象异构体为 TTTT 链段。1554 和 $1310 \mathrm{~cm}^{-1}$ 峰被分别指认为全同 $\mathrm{CH}_{2}$ 剪切振动和全 同 $\mathrm{CH}_{2}$ 扭转振动。

(TTG) $)_{10}$ 构象异构体为 TGTT 链段。 $1548 \mathrm{~cm}^{-1}$ 为 $\alpha / \beta^{\prime}+\alpha^{\prime} / \beta$ 两两同向剪切振动; $1519 \mathrm{~cm}^{-1}$ 为 $\alpha / \beta+\alpha^{\prime} / \beta^{\prime}$ 两两同向 $\mathrm{CH}_{2}$ 剪切振动; $1330 \mathrm{~cm}^{-1}$ 为 $\alpha / \beta+\alpha^{\prime} / \beta^{\prime}$ 两两同向 $\mathrm{CH}_{2}$ 扭转振动。

$(\mathrm{GTG})_{10}$ 构象异构体为 TGGT 链段。 $1536 \mathrm{~cm}^{-1}$ 为 $\alpha / \beta / \alpha^{\prime}$ 同向 $\mathrm{CH}_{2}$ 剪切振动; $1519 \mathrm{~cm}^{-1}$ 为 $\beta / \alpha^{\prime} / \beta^{\prime}$ 同 向 $\mathrm{CH}_{2}$ 剪切振动; $1355 \mathrm{~cm}^{-1}$ 为 $\alpha / \beta+\alpha^{\prime} / \beta^{\prime}$ 两两同向 $\mathrm{CH}_{2}$ 扭转振动。

(GTT-TTT-TTG-TTT) ${ }_{2}$-GTT-TTT 构象异构体为 TTTT、TGTT 和 TGGT 链段。 $1555 \mathrm{~cm}^{-1}$ 峰为 TTTT
链段的全同 $\mathrm{CH}_{2}$ 剪切振动; $1523 \mathrm{~cm}^{-1}$ 峰为 TGTT 链 段中 $\mathrm{G}$ 左边 $\alpha / \beta$ 同向 $\mathrm{CH}_{2}$ 剪切振动; $1544 \mathrm{~cm}^{-1}$ 峰为 TTTT(主要) 和 TGTT 两种链段的 $\mathrm{CH}_{2}$ 剪切振动; $1330 \mathrm{~cm}^{-1}$ 峰为 TGTT 链段中 $\mathrm{G}$ 左边 $\alpha / \beta$ 同向 $\mathrm{CH}_{2}$ 扭 转振动; $1312 \mathrm{~cm}^{-1}$ 峰为 TTTT 链段的全同 $\mathrm{CH}_{2}$ 扭转 振动。

$(\mathrm{GTT})_{2}(\mathrm{TTG})_{2}(\mathrm{GTT})_{2}(\mathrm{TTG})_{2}(\mathrm{GTT})_{2}$ 构象异构体 为 TGGT、TGTT 和 TTTT 链段。 $1558 \mathrm{~cm}^{-1}$ 峰为 TTTT 链段的全同 $\mathrm{CH}_{2}$ 剪切振动, 与 (GTT-TTTTTG-TTT $)_{2}$-GTT-TTT 构象异构体的 $1555 \mathrm{~cm}^{-1}$ 峰的 振动模式相同; $1536 \mathrm{~cm}^{-1}$ 峰为 TGGT 链段的全同 $\mathrm{CH}_{2}$ 剪切振动; $1524 \mathrm{~cm}^{-1}$ 峰为 TGTT 链段 $\mathrm{G}$ 型 $\mathrm{C}-$ $\mathrm{O}$ 键左边 $\alpha / \beta$ 同向 $\mathrm{CH}_{2}$ 剪切振动, 与 (GTT- TTTTTG-TTT $)_{2}$-GTT-TTT 构象异构体的 $1523 \mathrm{~cm}^{-1}$ 峰的 振动模式相同; $1344 \mathrm{~cm}^{-1}$ 峰为分子主链末端 TGGT 链段的 $\alpha / \beta+\alpha^{\prime} / \beta^{\prime}$ 两两同向 $\mathrm{CH}_{2}$ 扭转振动。1328 $\mathrm{cm}^{-1}$ 峰为 TTTT 链段的全同 $\mathrm{CH}_{2}$ 扭转振动。

$(\mathrm{GTG})_{6}(\mathrm{GTT})_{2}(\mathrm{GTG})_{2}$ 构象异构体为 TGGT、 TGTT 和 TTTT 链段。 $1536 \mathrm{~cm}^{-1}$ 峰为 TGGT 链段的 全同 $\mathrm{CH}_{2}$ 剪切振动, 与 $(\mathrm{GTT})_{2}(\mathrm{TTG})_{2}(\mathrm{GTT})_{2}(\mathrm{TTG})_{2}$ $(\mathrm{GTT})_{2}$ 构象异构体的 $1536 \mathrm{~cm}^{-1}$ 峰的振动模式相

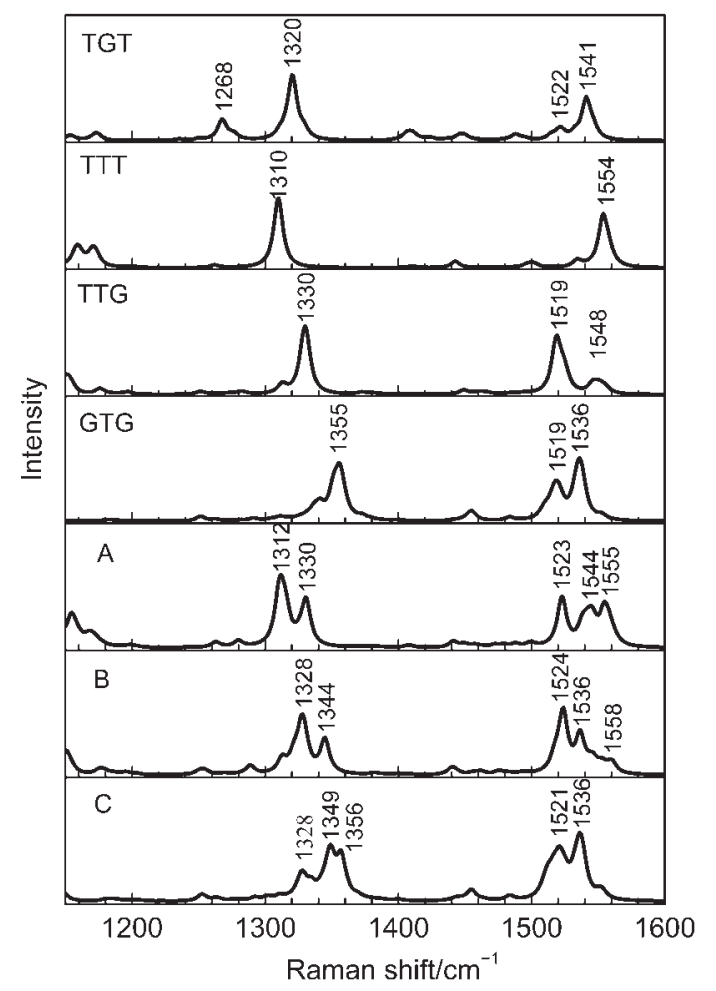

图 57 种 PEO400 构象异构体的 $1300-1600 \mathrm{~cm}^{-1}$ 区域的 计算拉曼光谱

Fig.5 Calculated Raman spectra of seven PEO400 conformations in $1300-1600 \mathrm{~cm}^{-1}$ region 


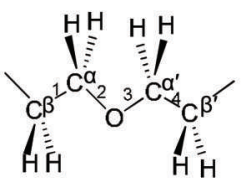

TTTT

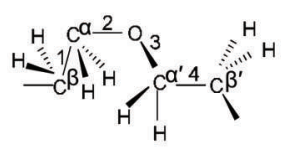

TGTT

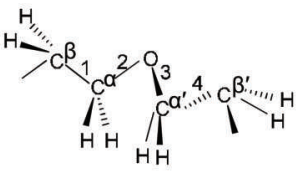

TGGT

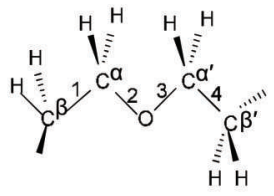

GTTG

图 6 与描述 $\mathrm{CH}_{2}$ 剪切振动和 $\mathrm{CH}_{2}$ 扭转振动相关的 4 种 $\mathrm{CH}_{2} \mathrm{CH}_{2}-\mathrm{O}-\mathrm{CH}_{2} \mathrm{CH}_{2}$ 构象

Fig.6 Four $\mathrm{CH}_{2} \mathrm{CH}_{2}-\mathrm{O}-\mathrm{CH}_{2} \mathrm{CH}_{2}$ conformations associated with the description of $\mathrm{CH}_{2}$ scissor and $\mathrm{CH}_{2}$ twist vibrations

同。 $1521 \mathrm{~cm}^{-1}$ 峰为 TGTT 链段的 $\mathrm{CH}_{2}$ 剪切振动; $1349 \mathrm{~cm}^{-1}$ 为 TGGT 链段的 $\alpha / \beta+\alpha^{\prime} / \beta^{\prime}$ 两两同向 $\mathrm{CH}_{2}$ 扭转振动, 与 $(\mathrm{GTT})_{2}(\mathrm{TTG})_{2}(\mathrm{GTT})_{2}(\mathrm{TTG})_{2}(\mathrm{GTT})_{2}$ 构 象异构体的 $1344 \mathrm{~cm}^{-1}$ 峰的振动模式相同; 1328 $\mathrm{cm}^{-1}$ 峰为 TGTT 链段的 $\mathrm{CH}_{2}$ 扭转振动; $1356 \mathrm{~cm}^{-1}$ 峰 为分子主链中部 TGGT 链段的 $\alpha / \beta+\alpha^{\prime} / \beta^{\prime}$ 两两同向 $\mathrm{CH}_{2}$ 扭转振动。

基于上述振动指认，我们注意到振动频率大 小与 $\mathrm{CH}_{2} \mathrm{CH}_{2}-\mathrm{O}-\mathrm{CH}_{2} \mathrm{CH}_{2}$ 链段构象的振动模式密 切相关。其频率大小顺序可归纳为。

剪切振动频率: TTTT 链段全同 $\mathrm{CH}_{2}$ 剪切振动 频率 $\left(1554-1558 \mathrm{~cm}^{-1}\right)>$ GTTG 链段的 $\alpha / \alpha^{\prime}+\beta / \beta^{\prime}$ 两 两同向 $\mathrm{CH}_{2}$ 剪切振动频率 $\left(1541-1548 \mathrm{~cm}^{-1}\right)>$ TGGT 链段的全同 $\mathrm{CH}_{2}$ 剪切振动频率 $\left(1536 \mathrm{~cm}^{-1}\right)>$ GTTG 链段的 $\alpha / \beta+\alpha^{\prime} / \beta^{\prime}$ 两两同向 $\mathrm{CH}_{2}$ 剪切振动频 率，或者 TGTT 链段 $\mathrm{G}$ 型 $\mathrm{C}-\mathrm{O}$ 键左边 $\mathrm{C}_{\alpha} \mathrm{H}_{2} / \mathrm{C}_{\beta} \mathrm{H}_{2}$ 同 向剪切振动频率 $\left(1519-1524 \mathrm{~cm}^{-1}\right)$ 。

扭转振动频率: 链中部 TGGT 链段的 $\alpha / \beta+\alpha^{\prime} /$ $\beta^{\prime}$ 两两同向 $\mathrm{CH}_{2}$ 扭转振动 $\left(1355-1356 \mathrm{~cm}^{-1}\right)>$ 分子 主链末端 TGGT 链段的 $\alpha / \beta+\alpha^{\prime} / \beta^{\prime}$ 两两同向 $\mathrm{CH}_{2}$ 扭 转振动 $\left(1344 \mathrm{~cm}^{-1}\right)$, 或者 TGGT 链段的 $\alpha / \beta+\alpha^{\prime} / \beta^{\prime}$ 两两同向 $\mathrm{CH}_{2}$ 扭转振动 $\left(1349 \mathrm{~cm}^{-1}\right)>\mathrm{TGTT}$ 链段 $\mathrm{G}$ 左边 $\alpha / \beta$ 同向 $\mathrm{CH}_{2}$ 同向扭转振动, 或 $\alpha / \beta+\alpha^{\prime} / \beta^{\prime}$ 两两 同向 $\mathrm{CH}_{2}$ 扭转振动 $\left(1330 \mathrm{~cm}^{-1}\right)$, 或 TGTT 链段的 $\mathrm{CH}_{2}$ 扭转振动 $\left(1328 \mathrm{~cm}^{-1}\right)>\mathrm{GTTG}$ 链段的 $\alpha / \alpha^{\prime}+\beta / \beta^{\prime}$ 两两同向 $\mathrm{CH}_{2}$ 扭转振动 $\left(1320 \mathrm{~cm}^{-1}\right)>\mathrm{TTTT}$ 链段的 全同 $\mathrm{CH}_{2}$ 扭转振动 $\left(1310-1312 \mathrm{~cm}^{-1}\right)>\mathrm{GTTG}$ 链段 的 $\alpha / \beta+\alpha^{\prime} / \beta^{\prime}$ 两两同向 $\mathrm{CH}_{2}$ 扭转振动 $\left(1268 \mathrm{~cm}^{-1}\right)$ 。

基于上述特征链段的振动频率分析, 参照基 于 (TGT) $)_{10}$ 异构体的实际拉曼光谱的初步指认, 对 图 4 中 1296 和 $1446 \mathrm{~cm}^{-1}$ 等谱峰进行了归属, 并对 各构象异构体的特征链段的贡献进行了探讨。

首先, 实验拉曼光谱中 $1468 \mathrm{~cm}^{-1}$ 拉曼峰被指 认为(TGT) ${ }_{10}$ 异构体 GTTG 链段的 $\alpha / \alpha^{\prime}+\beta / \beta^{\prime}$ 两两同 向 $\mathrm{CH}_{2}$ 剪切振动。因为 TTTT 链段全同 $\mathrm{CH}_{2}$ 剪切振 动频率 $\left(1554-1558 \mathrm{~cm}^{-1}\right)>$ GTTG 链段的 $\alpha / \alpha^{\prime}+\beta / \beta^{\prime}$
两两同向 $\mathrm{CH}_{2}$ 剪切振动频率 $\left(1541-1548 \mathrm{~cm}^{-1}\right)$, 故 $1468 \mathrm{~cm}^{-1}$ 峰右侧 (高频侧) 的部分强度被归属为 (TTT) 10 等构象异构体的 TTTT 链段的全同 $\mathrm{CH}_{2}$ 剪切 振动。我们注意到, 纯液态时 $1468 \mathrm{~cm}^{-1}$ 拉曼峰右 侧半峰宽较水溶液时大 $4 \mathrm{~cm}^{-1}$, 这说明(TTT $)_{10}$ 构 象异构体和各异构体 TTTT 链段在纯液态时有明显 的布居。水的存在不利于 TTTT 链段的布局。同 理, $1446 \mathrm{~cm}^{-1}$ 峰被归属为各异构体 TGTT 链段的 $\mathrm{CH}_{2}$ 剪切振动。

其次, 因实验拉曼光谱中 $1280 \mathrm{~cm}^{-1}$ 峰被指认 为 $(\mathrm{TGT})_{10}$ 异构体 GTTG 链段的 $\alpha / \alpha^{\prime}+\beta / \beta^{\prime}$ 两两同向 $\mathrm{CH}_{2}$ 扭转振动 $\left(1320 \mathrm{~cm}^{-1}\right)$, 故实验拉曼光谱中 1296 $\mathrm{cm}^{-1}$ 被指认为(TTG) ${ }_{10}$ 异构体 TGTT 链段的 $\alpha / \beta+\alpha^{\prime} /$ $\beta^{\prime}$ 两两同向 $\mathrm{CH}_{2}$ 扭转振动(计算频率 $1330 \mathrm{~cm}^{-1}$ )(主 要)。(GTT-TTT-TTG-TTT) ${ }_{2}$-GTT-TTT 异构体 TGTT 链段的 $\alpha / \beta$ 同向 $\mathrm{CH}_{2}$ 扭转振动(计算频率 $1330 \mathrm{~cm}^{-1}$ ) 和 $(\mathrm{GTG})_{6}(\mathrm{GTT})_{2}(\mathrm{GTG})_{2}$ 构象异构体 TGTT 链段的的 $\mathrm{CH}_{2}$ 扭转振动(计算频率 $1328 \mathrm{~cm}^{-1}$ )对 $1296 \mathrm{~cm}^{-1}$ 峰也 有一定的贡献。概括起来, $1296 \mathrm{~cm}^{-1}$ 峰可归属为 各异构体 TGTT 链段的 $\mathrm{CH}_{2}$ 扭转振动。

\section{4 结 论}

本文采用密度泛函理论计算方法结合红外和 拉曼光谱实验手段研究了常温液态下 PEO400 构象 异构体的结构和振动光谱。基于 6 种 $\mathrm{EO}$ 重复单元 构象的定义，构建并优化得到了 4 种全同 EO构象 组合 $(\mathrm{TGT})_{10}$ 、 $(\mathrm{TTT})_{10}$ 、(TTG) $)_{10}$ 和 $(\mathrm{GTG})_{10}$, 以及 3 种其它 EO 构象组合的 PEO400 构象异构体, 并在 PCM 溶剂模型和 B $3 L Y P / 6-31 \mathrm{G}(d)$ 理论水平下获得 了它们的振动光谱, 重点开展了 $\mathrm{CH}_{2}$ 剪切振动和 $\mathrm{CH}_{2}$ 扭转振动的模式分析, 并将结果应用于液态和 水溶液中的拉曼光谱指认。本文发现, 决定 $\mathrm{CH}_{2}$ 剪 式振动和 $\mathrm{CH}_{2}$ 扭转振动频率大小的关键因素是 $\mathrm{CH}_{2} \mathrm{CH}_{2}-\mathrm{O}-\mathrm{CH}_{2} \mathrm{CH}_{2}$ 链段的构象及其振动模式, 当 $(\mathrm{TGT})_{10}$ 被确定为主要成分时, 只要 $\mathrm{CH}_{2} \mathrm{CH}_{2}-$ $\mathrm{O}-\mathrm{CH}_{2} \mathrm{CH}_{2}$ 链段的构象及其振动模式穷尽了, 就 
可避开与其它 EO 构象组合相关的重要构象异构体 数量穷尽的难题。具体结论如下:

(1) 无论是 4 种全同 EO 构象组合 (TGT) 10 、 $(\mathrm{TTT})_{10}$ 、 $(\mathrm{TTG})_{10}$ 和 $(\mathrm{GTG})_{10}$, 还是其它任意 $\mathrm{EO}$ 构 象组合的构象异构体, 其 $\mathrm{CH}_{2}$ 剪切振动和 $\mathrm{CH}_{2}$ 扭转 振动模式均与 $\mathrm{CH}_{2} \mathrm{CH}_{2}-\mathrm{O}-\mathrm{CH}_{2} \mathrm{CH}_{2}$ 链段的 TTTT、TGTT、TGGT 和 GTTG 构象相关。

(2) $\mathrm{CH}_{2}$ 剪切振动和扭转振动频率大小由链段 的构象决定:

(i) TTTT 链段全同 $\mathrm{CH}_{2}$ 剪切振动 $>$ GTTG 链段 $\alpha / \alpha^{\prime}+\beta / \beta^{\prime}$ 两两同向 $\mathrm{CH}_{2}$ 剪切振动频率(1541-1548 $\left.\mathrm{cm}^{-1}\right)>$ TGGT 链段的全同 $\mathrm{CH}_{2}$ 剪切振动频率 (1536 $\left.\mathrm{cm}^{-1}\right)>$ GTTG 链段的 $\alpha / \beta+\alpha^{\prime} / \beta^{\prime}$ 两两同向 $\mathrm{CH}_{2}$ 剪切 振动频率(1519-1524 $\left.\mathrm{cm}^{-1}\right)$;

(ii) 主链中部 TGGT 链段的 $\alpha / \beta+\alpha^{\prime} / \beta^{\prime}$ 两两同向 $\mathrm{CH}_{2}$ 扭转振动 $\left(1355-1356 \mathrm{~cm}^{-1}\right)>$ 主链末端 TGGT 链段的 $\alpha / \beta+\alpha^{\prime} / \beta^{\prime}$ 两两同向 $\mathrm{CH}_{2}$ 扭转振动 (1344$\left.1349 \mathrm{~cm}^{-1}\right)>$ TGTT $\alpha / \beta+\alpha^{\prime} / \beta^{\prime}$ 两两同向 $\mathrm{CH}_{2}$ 扭转振 动 $\left(1330 \mathrm{~cm}^{-1}\right)>\mathrm{GTTG}$ 的 $\alpha / \alpha^{\prime}+\beta / \beta^{\prime}$ 两两同向 $\mathrm{CH}_{2}$ 扭 转振动 $\left(1320 \mathrm{~cm}^{-1}\right)>$ TTTT 链段的全同 $\mathrm{CH}_{2}$ 扭转振 动 $\left(1310-1312 \mathrm{~cm}^{-1}\right)>$ GTTG 的 $\alpha / \beta+\alpha^{\prime} / \beta^{\prime}$ 两两同向 $\mathrm{CH}_{2}$ 扭转振动 $\left(1268 \mathrm{~cm}^{-1}\right)$ 。

(3) $1296 \mathrm{~cm}^{-1}$ 峰可归属为各异构体 TGTT 链段 的 $\mathrm{CH}_{2}$ 扭转振动; $1446 \mathrm{~cm}^{-1}$ 峰可归属为各异构体 TGTT 链段的 $\mathrm{CH}_{2}$ 剪切振动; $1468 \mathrm{~cm}^{-1}$ 峰右侧 (高 频侧)的部分强度被归属各异构体 TTTT 链段的全 同 $\mathrm{CH}_{2}$ 剪切振动。

\section{References}

(1) Miyazawa, T.; Fukushima, K.; Ideguchi, Y. J. Chem. Phys. 1962, 37, 2764. doi: 10.1063/1.1733103

(2) Yoshihara, T.; Tadokoro, H.; Murahashi, S. J. Chem. Phys. 1964, 41, 2902. doi: 10.1063/1.1726373

(3) Koenig, J. L.; Angood, A. C. J. Polym. Sci. A 1970, 8, 1787.

(4) Tadokoro, H.; Chatani, Y.; Yoshihara, T.; Tarahara, S.; Murahashi, S. Makromol. Chem. 1964, 73, 109.

(5) Matsuura, H.; Miyazawa, T. J. Polym. Sci. A 1969, 7, 1735.

(6) Takahashi, Y.; Tadokoro, H. Macromolecules 1973, 6, 673.

(7) Matsuura, H.; Miyazawa, T. Bull. Chem. Soc. Jap. 1968, 41, 1798.

(8) Yakoyama, M.; Ochi, H.; Tadokoro, H.; Price, C. Macromolecules 1972, 5, 690.

(9) Rabolt, J. F.; Johnson, K. W.; Zitter, R. N. J. Chem. Phys. 1974, 61, 504. doi: 10.1063/1.1681924

(10) Migliardo, F.; Magazù, S.; Caccamo, M. T. J. Mol. Struct. 2013, 1048, 261. doi: 10.1016/j.molstruc.2013.05.060

(11) Chaurasia, S. K.; Singh, R. K.; Chandra, S. Vibrational Spectroscopy 2013, 68, 190. doi: 10.1016/j.vibspec.2013.08.001

(12) Frech, R.; Huang, W. Macromolecules 1995, 28, 1246.

(13) Chatani, Y.; Fujii, Y.; Takayanagi, T.; Homma, A. Polymer 1990, 31, 2238.

(14) Chatani, Y.; Okamura, S. Polymer 1987, 28, 1815.

(15) Iwamoto, R.; Saito, Y.; Ishihara, H.; Tadokoro, H. J. Polym. Sci. Polym. Phys. Ed. 1968, 6, 1509.

(16) Yokoyama, M.; Ishihara, H.; Iwamoto, R.; Tadokoro, H.; Macromolecules 1969, 2, 184.

(17) Paoke, B. L.; Ratner, M. A.; Shriver, D. F. J. Phys. Chem. Solids 1981, 42, 493.

(18) Shephard, J. J.; Bremer, P. J.; McQuillan, A. J. J. Phys. Chem. B 2009, 113, 14229. doi: 10.1021/jp905149z 